\title{
Controllability of Flow-Conservation Transportation Networks with Fractional-Order Dynamics
}

\author{
Wei Chen (iD) and Bo Zhou \\ College of Mathematics and Statistics, Chongqing Jiaotong University, Chongqing 400074, China \\ Correspondence should be addressed to Bo Zhou; bzhou@cqjtu.edu.cn
}

Received 12 August 2021; Accepted 3 November 2021; Published 23 November 2021

Academic Editor: Thach Ngoc Dinh

Copyright ( $\odot 2021$ Wei Chen and Bo Zhou. This is an open access article distributed under the Creative Commons Attribution License, which permits unrestricted use, distribution, and reproduction in any medium, provided the original work is properly cited.

In this paper, we adapt the fractional derivative approach to formulate the flow-conservation transportation networks, which consider the propagation dynamics and the users' behaviors in terms of route choices. We then investigate the controllability of the fractional-order transportation networks by employing the Popov-Belevitch-Hautus rank condition and the QR decomposition algorithm. Furthermore, we provide the exact solutions for the full controllability pricing controller location problem, which includes where to locate the controllers and how many controllers are required at the location positions. Finally, we illustrate two numerical examples to validate the theoretical analysis.

\section{Introduction}

1.1. Motivations. Recent years have witnessed the explosive growth of motorized vehicles in transportation networks, which leads the transportation networks to be more and more congested in serious traffic safety problems. So, the transportation networks require control. In practice, the control instruments, for instance, the traffic lights, the ramps, and the pricing controllers, are often installed into the key nodes in the transportation networks, so as to make the transportation networks operate smoothly.

In order to assess whether the controlled transportation networks are capable of reaching the desired performance, the concept of "controllability" is introduced, which is originated from the control theoretical concept of complex dynamical networks. A complex dynamical network is said to be controllable, if any other state can be reached from its current state through a given control action. The research of controllability of integer-order complex networks has been a hot topic [1-5]. From a transportation networks' perspective, for example, whether the congestion can be eased via the first-best pricing controller on the links is actually a controllability problem. Up till now, there are some existing works that investigate the controllability of transportation problem, such as the traffic signal timing plan and the dynamics equilibrium based on traffic control, which has been discussed in [6,7]; the relationship between route choice and traffic control has been discussed in [8]; the Gramian-based optimization analysis of the traffic control problem is considered in [9]; the route choice and controller problem in transportation has been clear expressed in [10]. The above dynamics are represented by integer-order dynamics system. In practice, however, the fractional-order systems outperform the integer-order systems, because they are more effective to describe the memory and heredity of dynamical systems, which are two essential characteristics and behaviors of the real transportation networks. Because of the advantages provided by the fractional-order dynamical systems, it has found wide applications but rarely mentioned in the controllability of transportation networks. Thus, we aim to fill this gap in this paper. Fractional-order systems can provide natural frameworks for modeling of many practical dynamical systems, such as economic systems [11] and variable structure hybrid energy storage system [12]. The Caputo derivatives allow traditional, initial, and boundary conditions to be included in the formulation of the considered problem; its application to engineering and modeling of complex networks attracted much attention in 
recent years. The stability of nonlinear Caputo fractionalorder systems with control vector was investigated in $[11,13,14]$, and the stability of $2 \mathrm{D}$ nonlinear fractionalorder systems was discussed in [15]. The numerical approximation for the expansion of Caputo fractional-order nonlinear systems was studied in $[16,17]$. Controllability is one of the important issues in the study of fractional-order systems; the controllability of Caputo fractional-order complex networks is discussed in [18-20]. Transportation networks are typical complex networks, so we introduce the Caputo fractional derivative to describe the dynamics of the fractional-order transportation networks. In the existing controllability research works, the Kalman rank condition is widely utilized. Comparing with Kalman rank condition, the Popov-Belevitch-Hautus ( $\mathrm{PBH}$ ) rank condition has the following advantages: Firstly, it is suitable for any directed or undirected network structure, with or without link weight and self-circulation [21]; secondly, it can analyze the controllability of multiplex networks using multiple-relation networks and multiple-layer networks with interlayer couplings as two classes of prototypical systems [22]. Due to the complexity nature of the fractional-order transportation networks, we should first obtain the minimum number of controller nodes and then check whether the $\mathrm{PBH}$ rank condition is satisfied. There is a problem of insufficient accuracy in solving eigenvalue of characteristic equation with fractional-order transportation networks, and the QR decomposition algorithm because it does not miss any eigenvalues, backward stability requiring less steps is the best algorithm for asymmetric matrices. In this work, we investigate the problem of controllability for transportation networks with fractional derivatives. Then, we propose a simple control method using the $\mathrm{PBH}$ rank condition and the QR to get the specific number and installation position. In Section 2, system model and the main results are obtained. In Section 3, two numerical examples are presented to demonstrate the effectiveness of the proposed results. Conclusions are presented in Section 4.

1.2. Literature Review. Controllability is an important concept in modern control theory. If we can choose the minimum number of the driver nodes and switch the system from any initial state to the desire final state, the dynamic system is controllable. In [23], the author proposed the Kalman rank condition to choose the driver nodes and made a preparation for the problem of controllability of linear dynamic systems. As the work in [24], an algorithm inspired by the Kalman controllability rank condition is mentioned to study the control scheme of complex networks in infinite time. In [21], the PBH rank condition can be introduced to make up for the deficiency of Kalman rank condition; for the controllability of arbitrary structure and link weight network, the controllability mainly depends on the connectivity of network nodes, instead of the exact value of network nodes.

Since then, the research on the controllability of complex networks has been a major consideration in many research articles. In [22], a framework is developed to analyze the controllability of multiple networks using multiple-relation networks and multiple-layer networks with interlayer couplings. Based on the strong structural characteristics of controllability and observability, the simple greedy heuristics algorithm that maximizes the rank of the controllable matrix can achieve the effective global optimization in [25]. In [26], the problem for NP-hard minimum driver nodes is investigated, which utilizes the simple greedy heuristic algorithm to find the minimum driver nodes. In [27], the effect of degree distribution to the controllability for the considered networks is analyzed. In [28], the model about physical controllability is proposed, which considers the costs caused by calculation error in actual systems. In [29], the author devises an algorithm that selects the minimum controls from a given collection of inputs in polynomial time.

The transportation networks are typical complex networks due to their complex internal nodes, numerous subsystems, and complex hierarchical structure. Additionally, the controllability plays a vital role in the transportation networks. In [30], the author first introduced a methodology for devising optimal green splits for isolated intersections, based on their topological characteristics and the vehicular flows. In [31], the problem of congestion in different parts of the networks is discussed, whose the key factors including the departure time, route choice, and mode selection may cause or aggravate congestion. Therefore, we take much consideration on the related factors such as route choice in the controllability of transportation networks.

The increasing number of motor vehicles and the limit of urban road design have caused traffic congestion seriously. There are two effective ways to control traffic congestion, one is to increase infrastructure construction, and the other is to carry out effective traffic management and control. However, the development of urban transportation infrastructure has reached a level of saturation, so the improved method is to develop effective traffic control strategies. In the early 1980s, the coordinated traffic control strategies were introduced to align the configurations of multiple controllers in a given network. Further research introduced by the explicit traffic model on the coordinated control strategies has aroused much attention. The works in [32] established the transportation networks model, which considered the design of traffic lights and the choice of user routes.

A solution technique based on the iterative optimization and assignment method is proposed in [7], which takes it to solve the combined dynamic user equilibrium and signal control problem. On the basis of the complexity of traffic models, the schemes with high efficiency and fast calculation have been put forward successively, such as model predictive control. In [33], the conservation of vehicles equation is considered to estimate the highway traffic flow. In [34], the characteristics of residents' travel are considered to estimate urban traffic state. In [35], a Bayesian probabilistic model to estimate traffic states is proposed to fuse all data to estimate urban traffic state. In [36], the stochastic hybrid model is considered to estimate urban traffic flow. A similar trend in the resulting complexity of the related prediction model can also be observed, as the work in [37-39]. 
In the study of the controllability of transportation networks, the design of appropriate control strategies that avoid or alleviate congestion plays an important role. In [40], urban transportation networks are modeled as a discretetime state-space model, and the traffic response strategy under state and control constraints is constructed to avoid congestion by using the $H_{\infty}$ control method and linear matrix inequality. Bianchin and Pasqualetti [9] mentioned a simplified version of the classic model of urban transportation networks and studied the optimization problem about the overall efficiency of the network by controlling the green light duration of intersections under current congestion conditions. In [10], the connection between the firstorder traffic flow theory and the structural control theory is established, which considered the congestion formation, propagation, and dissipation, thereby generating a solid modeling framework. In this framework, we can apply the existing controllability theories and algorithms to give an accurate solution to the transportation networks controller position problem.

Compared with the above-mentioned literature, most of the research on transportation networks is based on integer order, and the research on fractional order is very few. Fractional-order calculus can be regarded as an extension of classical calculus from integer order to any order. The main advantage is that it can describe the memory and heritability of the system and better reveal the essential characteristics of the system. Therefore, compared with integer-order calculus, the related fractional-order calculus model is widely applied in science and engineering $[12,41]$. Recently, the research of fractional-order systems has been a hot topic, as the work in $[16,17,42,43]$. The problem of stability with fractional-order system, for example, has been studied in $[11,13]$. In $[18,19]$, the controllability of complex networks is extended from integer order to fractional order, which shows that the control theory is applicable to fractional-order complex networks. In reality, many dynamic systems cannot be accurately described by a linear system, so the study of nonlinear systems is indispensable. A set of sufficient conditions that study the controllability study of the first-order nonlinear fractionalorder dynamic system are established in [20].

The fractional-order systems more effectively describe the memory and inheritance of dynamic systems. The control strategy and the flow estimation are rarely mentioned in the controllability of the transportation networks based on the fractional-order dynamics. Therefore, there are some difficulties in the selection of fractional-order operators and the controller strategy of fractional-order transportation networks. Mathematicians have defined a series of classical fractional derivatives from different angles and application requirements. Commonly used definitions for fractional derivatives are Riemann-Liouville and Caputo. The Caputo and the extension of Caputo fractional derivative are often used in actual engineering modeling. Making a comparison with the existing results, the classical Caputo derivatives because they allow traditional initial and boundary conditions to be included in the formulation of the considered problem were used to model the fractional-order transportation networks in this paper.
The specific research on the location and number of controllers is rarely considered as a major factor of the control strategies, in the literature of traffic control network. The controllability of fractional-order transportation networks and its determination of the position and number of controllers have become a major difficulty. The traditional Kalman rank condition is sufficient to judge the controllability of fractional-order complex networks, but there are obvious problems when using the Kalman rank condition to determine the controller of fractional-order transportation networks. Most of the existing literatures on controllability are based on Kalman condition, but PBH rank condition has great advantages in dealing with the controller problem of fractional-order transportation networks because it does not need to consider the weight of nodes but only considers whether nodes are connected. This paper mainly studies the controllability of the fractional-order transportation networks with the PBH rank condition.

Motivated by the above discussion, the major contributions of this paper are threefold:

(1) We introduce the Caputo fractional derivative to describe the dynamics of the flow-conservation transportation networks, which allow for traditional initial and boundary conditions. Compared with the integer-order system, it can better describe the selforganization and dynamic behavior of the transportation networks.

(2) We employ the PBH rank condition to convert the fractional-order transportation networks controllability problem into an eigenvalue problem, which indicates that the minimum number of drive nodes in the fractional-order transportation networks is equal to the maximum geometric multiplicity of the incidence matrix eigenvalues.

(3) We utilize the QR decomposition algorithm to calculate the eigenvalues of the incidence matrix, so as to improve the problem of insufficient accuracy when solving eigenvalues by the characteristic equation method. In addition, the QR decomposition algorithm has backward stability and local quadratic convergence, so that fewer steps are required to solve the eigenvalues.

\section{Methods}

This section outlines the steps taken to adapt the structural controllability theory of complex networks with flowconservation to the specific conditions of fractional-order transportation networks. For clarity, at the beginning of this section, we briefly reviewed the controllability, main assumptions, and general formulas of the flow-conservation complex networks. Then, we explained that the classical fractional-order derivative is introduced into the framework of flow-conservation controllability and deduced it to the dynamics of the fractional-order transportation networks, as well as the formulation of the dynamics of standard traffic control supporting infrastructure tools. 
2.1. Flow-Conservation Complex Networks Control Theory. To introduce the concepts of flow-conservation complex networks control theory [44] and its application to fractional-order transportation networks, we begin by the dynamical equations of a linear time-invariant control system associated with flow-conservation dynamics as

$$
X(k)=W \cdot X(k-1)+B \cdot U(k-1) .
$$

In the specific example of a flow-conservation complex network, $X$ is the state vector that captures the flow states of $N$ nodes, dependent on the current time $K$. The element $W_{i j}$ of the flow transfer matrix $W$ denotes the link weight of $i$ associated with its neighbors $j$; $W_{i j}=0$ means that node $i$ has no flow moving to node $j . U$ is the vector of $m$ controllers with $U=\left(u_{1}, u_{2}, \ldots, u_{m}\right)$, and $B$ is the $N \times m$ control matrix.

The controllability of the flow-conservation complex networks is based on the exact control theory [21], which shows that the system is fully controllable if and only if

$$
\operatorname{rank}\left(\mathrm{cI}_{N}-w, B\right)=N \text {, }
$$

is satisfied for any complex number $c$, where $I_{N}$ is the identity matrix of dimension $N$. There are many possible control matrices $B$ that satisfy the controllable $\mathrm{PBH}$ rank condition. The most important goal is to find a set of $B$ corresponding to the minimum number $N_{D}$ of independent controllers required to control the whole network.

The controllability of network with flow-conservation dynamics can be considered by just analyzing the network flow transfer matrix $W$. The minimum number $N_{D}$ of controllers is determined by the maximum geometric multiplicity $\mu\left(\lambda_{i}\right)$ of the eigenvalue $\lambda_{i}$ of $W$

$$
N_{D}=\max _{i}\left\{N-\operatorname{rank}\left(\lambda_{i}^{W} I_{N}-W\right)\right\},
$$

where the $\lambda_{i}^{W}$ represents the eigenvalue of matrix $W$.

Each node in the flow-conservation networks receives flows from other nodes through links. The number of flows on a link depends on the link weight. The state of the nodes will change according to the number of flows it receives and sends out. The total flows in the network can be regarded as a constant and can be used to approximately model transportation network. In the next section, we derive the assumptions and conditions upon which controllability of the fractional-order transportation networks can be successfully modeled through (1)-(3).

\subsection{Modeling of the Fractional-Order Transportation} Networks. In order to successfully modify the dynamics of the fractional-order transportation networks to the framework introduced in the previous section, a set of definitions and assumptions must be met. Firstly, flow-conservation networks control theory is based on integer order, but in this paper we apply the Caputo fractional derivative to the flowconservation networks to obtain the dynamic formulation of the fractional-order transportation networks. Mathematicians have defined a series of classical fractional derivatives from different angles and application requirements, where the Caputo fractional derivative is often used in actual engineering modeling which can simplify Laplace transform. The definition of Caputo derivative is

$$
{ }_{a} D_{t}^{\alpha}=\frac{1}{\Gamma(n-\alpha)_{a}} \int_{a}^{t}(t-\tau)^{n-\alpha-1} f^{(n)}(t-\tau) \mathrm{d} \tau,(n-1<\alpha<n),
$$

where $\Gamma[\cdot]$ is the Gamma function.

Remark 1. In this paper, the controllability of fractionalorder transportation networks is considered, where Caputo derivatives because they allow traditional initial and boundary conditions to be included in the formulation of the considered problem are discussed, which is the same with those in $[11,13,18]$. However, in $[16,17]$, the numerical method for solving the extension of Caputo derivatives of factional-order complex system was considered. The extension of Caputo derivatives in [16] is known to have both nonlocal and nonsingular kernels properties, and in [17] biorder contains power law and exponential decay.

Secondly, we consider that the fractional-order transportation networks are modeled by the first-order traffic flow theory method applicable to Newell's simplified motion wave theory. And users who entered the network have perfect information about the network travel time, choose the shortest route, and make the corresponding response which has been considered. Also, due to its linearity, the modeling framework is greatly simplified while still allowing the correct capture of congestion dynamics.

The discrete-time dynamics of fractional-order linear time-invariant dynamic system associated with flow-conservation dynamics [18] is

$$
D^{\alpha} X(k)=W \cdot X(k-1)+B \cdot U(k-1),
$$

where $D^{\alpha}$ is the operator for the Caputo fractional derivative of order $\alpha, 0<\alpha<1$. In this paper, the state vector $X(k)$ capturers the cumulative vehicle number of each node in the networks, dependent on the current time $k$. The matrix $W$ denotes the link weight. The matrix $B$ expresses the coupling between the $M$ controllers equipped on a network and the controlled nodes, called input matrix. $U(k)$ is the effect of controllers action on its controlled nodes.

We begin to derive the equation captures vehicle propagation dynamics; the small road node diagram of the universal fractional-order transportation networks is shown as in Figure 1(a). Figure 1(b) is a small network cutout of Figure 1(a), ignore the number of vehicles entering or outputting from other nodes at this time, and only consider the node 1 and node 2 . The cumulative vehicle number of nodes 2 follows first-order dynamics [10]; the equation can be shown as follows:

$$
D^{\alpha} X_{2}(k)=w_{1,2} X_{1}\left(k-t_{1,2}(k-1)\right),
$$

where the $t_{i, j}(k-1)$ represents the time difference of node $i$ and node $j$ reached the same number of vehicles. This value is related to the congestion level of the networks; in the case 


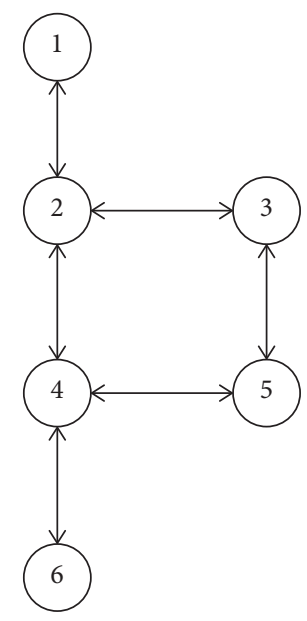

(a)

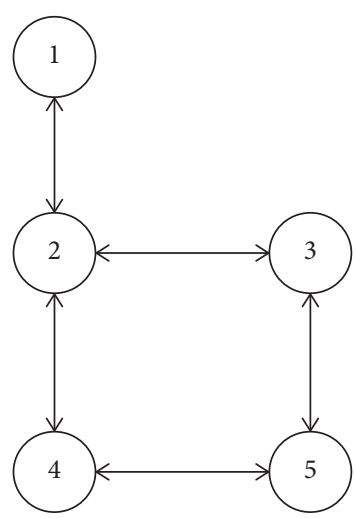

(b)

Figure 1: (a) A small cutout fractional-order transportation network with six nodes. (b) Five links networks cutout.

of congestion, the value will increase when the road is congested and vice versa.

The fractional-order transportation networks exhibit a certain degree of self-organization, so we have specially considered the influence of route choice. Specifically, we assume that the system operates according to the principle of deterministic user equilibrium; that is, we hope that each user will enter the system through choosing the cheapest route appropriately at that moment to minimize his/her own travel time. By introducing the probability distribution of the travel time variable $t_{i, j}(k)$, stochastic user equilibrium can also be considered.

Explicit consideration of route choice dynamics in the problem allows us to extend the above system dynamics. Consider the small network in Figure 1(b); the route from node 1 to node 5 has two possible routes. We can easily extract the following relationships between the state variables of nodes 3,4 , and 5 :

$$
\begin{aligned}
& D^{\alpha} X_{3}(k)=X_{2}\left(k-t_{2,3}(k-1)\right)-X_{4}\left(k-t_{2,3}(k-1)+t_{2,4}(k-1)\right), \\
& D^{\alpha} X_{4}(k)=X_{2}\left(k-t_{2,4}(k-1)\right)-X_{4}\left(k-t_{2,4}(k-1)+t_{2,3}(k-1)\right), \\
& D^{\alpha} X_{5}(k)=w_{3,5} X_{3}\left(k-t_{3,5}(k-1)\right)+w_{4,5} X_{4}\left(k-t_{4,5}(k-1)\right) .
\end{aligned}
$$

Remark 2. By applying the path flow-conservation effect at each node, the first two equations of (7) state that the sum of vehicles passing through nodes 3 and 4 at time $\mathrm{k}$ must be equal to the sum of vehicles passing through node 2 at the appropriate previous time. When only a certain route is used, the equation will collapse into simple node-to-node propagation dynamics.

So far, fractional-order networks dynamics can be summarized by the following equation:

$$
D^{\alpha} X(k)=W \cdot X(k-T) .
$$

The dynamics of (8) includes travel time dependence $T$, which represents the pure delay component. However, this dynamic cannot be directly captured within the framework of the controllability of a complex network with flowconservation. Moreover, it is more complicated to model any node in the network to obtain the appropriate weight value $w_{i, j}$. Based on this consideration, in order to evaluate the control possibility of the fractional-order transportation networks, we can assume that the vehicle flow characteristics of the fractional-order transportation networks are modeled by an effective conversion method:

$$
D^{\alpha} X(k)=A \cdot X(k-1),
$$

where the vector $A$ can be derived as follows:

$$
a_{i, j}(k)=w_{i, j}(k-1) \frac{X_{i}\left(k-t_{i, j}(k-1)\right)}{X_{i}(k)} .
$$

Remark 3. The element $a_{i, j}(k)$ of matrix $A$, except the time dynamic routing split information $w_{i, j}(k-1)$, and the ratio of preceding vehicle cumulative between time $\left(k-t_{i, j}(k-1)\right)$ and time $(k-1)$ of the node, are also captured. Therefore, this ratio can be viewed as the ratio of congestion. The smaller the coefficient, the more the crowd. $a_{i j}(k)=1$ means that the two-node road is unobstructed without congestion; for real complex networks, the ratio of congestion collapse to zero is rare, so $0<a_{i j}(k) \leq 1$. 
In addition to modeling the dynamics of vehicle propagation and route choice, in order to construct the controllable structure of fractional-order transportation networks, it is necessary to accurately capture the dynamics of various control support infrastructures of the above network operation. There are many controllers in the real traffic system, but this paper mainly discusses the pricing controller. Next, we mainly consider the example of Figure 2 and install pricing controller with the link between node 2 and node 4 . follows:

The dynamics equation on node 4 can be described as

$$
\begin{aligned}
D^{\alpha} X_{4}(k) & =\left[a_{2,4}-\varphi_{2,4}(k-1)\right] X_{2}(k-1), \\
\varphi_{2,4}(k) & =f\left(p_{2,4}(k)\right), \varphi_{2,4}(k) \in\left[-a_{2,4}, 0\right],
\end{aligned}
$$

where the $\varphi_{2,4}(k-1)$ represents the impact of the number of vehicles between two nodes after the link is installed with a controller. In other words, imposing a toll $p_{2,4}(k)$ on the link between nodes 2 and 4 will result in a reduction effect $\varphi_{2,4}(k)$ of the cumulative number of vehicles moving from node 2 to node 4 , which is a function of the toll level itself. So as to satisfy the structure required by (5), we should simplify (11) as

$$
D^{\alpha} X_{4}(k)=a_{2,4} \cdot X_{2}(k-1)-\varphi_{2,4}(k-1) \cdot X_{2}(k-1),
$$

where

$$
\begin{aligned}
b_{2,4} & =1, \\
U_{2}(k-1) & =-\varphi_{2,4}(k-1) \cdot X_{2}(k-1) .
\end{aligned}
$$

According to the above formula of route choice, road congestion, and pricing controller model, we can finally obtain the system as

$$
\begin{aligned}
D^{\alpha} X(k) & =A \cdot X(k-1)+B \cdot U(k-1), \\
A & =\left\{a_{i, j}(k)=\omega_{i, j}(k) \frac{X_{i}\left(k-t_{i, j}(k-1)\right)}{X_{i}(k)}\right\}, \\
B & =\left\{b_{i, j}=\{1,0\}\right\}, \\
U_{i}(k) & =-\varphi_{i, j}(k) \cdot X_{i}(k) .
\end{aligned}
$$

\subsection{Controllability of Fractional-Order Transportation} Networks. The controllability of the complex networks with flow-conservation and even the fractional-order transportation networks can be determined by the Popov-Belevitch-Hautus $(\mathrm{PBH})$ rank condition. It is saying that the system is fully controllable if and only if

$$
\operatorname{rank}\left(\mathrm{cI}_{N}-\mathrm{AB}\right)=N \text {. }
$$

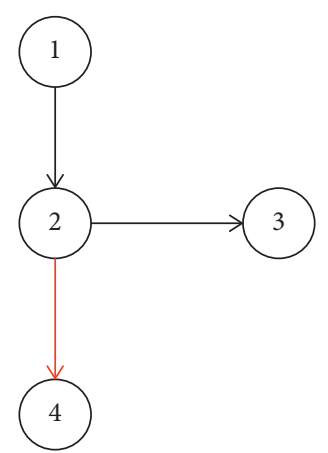

FIGURE 2: Four nodes cutout, installing pricing controller with the link between node 2 and node 4 .

Remark 4. For a linear time-invariant system, Kalman rank condition $\left[\begin{array}{lllll}B & \mathrm{AB} & A^{2} B & \ldots & A^{N-1} B\end{array}\right]=N$ is sufficient and necessary principle to ensure controllability [1]. But we cannot make a judgment through classical controllability theory in many cases, especially in the system with very large size or unknown parameters. The work in [21] introduces an exact controllability paradigm to identify the minimum set of driver nodes to achieve full control with arbitrary structures and link weight distributions.

The minimum number of driver nodes of $N_{D}$ is generally defined in terms of $B$ as $N_{D}=\min \{\operatorname{rank}(B)\}$. In one word, system controllability is to find the minimum number of driver nodes to satisfy this condition. So, to some extent, the $\mathrm{PBH}$ rank condition is equivalent to the Kalman rank condition.

The $\mathrm{PBH}$ condition proved that the minimum number of independent driver nodes is equal to the maximum geometric multiplicity of all eigenvalues of the network matrix, as

$$
N_{D}=\max _{i}\left\{\mu\left(\lambda_{i}\right)\right\}
$$

and $\mu\left(\lambda_{i}\right)$ is given as follows:

$$
\mu\left(\lambda_{i}\right)=\operatorname{dim} V_{\lambda_{i}}=N-\operatorname{rank}\left(\lambda_{i} I_{N}-A\right),
$$

where $\lambda_{i}$ represents the eigenvalue of matrix $A$. This condition transforms the network controllability problem into an eigenvalue problem, which greatly facilitates the analysis, provides a more comprehensive understanding of the network controllability, and is more suitable for the research of controllability of fractional-order transportation networks.

In the calculation of matrix eigenvalue problems, a simple idea is to solve the characteristic equation $\operatorname{det}\left(A-\lambda I_{N}\right)=0$. Because the coefficients of characteristic equation cannot be calculated by stable numerical method, even if the coefficient of characteristic equation can be accurately calculated, the root of characteristic polynomial $f(\lambda)=\operatorname{det}\left(A-\lambda I_{N}\right)$ may be particularly sensitive to the coefficients of polynomial under limited precision, and when the order of determinant is large, the calculation of 
determinant is large. This needs to find a more suitable way to solve the eigenvalue.

The typical methods to solve eigenvalues are power method, the QR algorithm, homotopy method, Lanczos method, Rayleigh algorithm, and so on. The most basic and simplest method is the power method, but for asymmetric matrix the power method only obtains the maximum eigenvalue of the matrix. In this paper, finding the maximum geometric multiplicity of matrix eigenvalue requires us to find all eigenvalues of matrix, so we need a method to find all eigenvalues. Here, all eigenvalues and eigenvectors of the matrix can be obtained by finite iterations using the $\mathrm{QR}$, and the results have rear stability; therefore the $\mathrm{QR}$ is chosen as the main method of fractional-order transportation networks controllability research. Next is a simple explanation of the QR.

For the matrix $A_{0}=A$, basic iterative scheme for the $\mathrm{QR}$ is

$$
\left\{\begin{array}{l}
A_{m-1}=Q_{m} \cdot R_{m} \\
A_{m}=R_{m} \cdot Q_{m}
\end{array}\right.
$$

where $Q_{m}$ is the unitary matrix and $R_{m}$ is the upper triangular matrix. It takes a lot of computations to complete an QR iteration, so we should make appropriate transformation before $\mathrm{QR}$ iteration to make the matrix have more zero elements. In this paper, we mainly use householder transformation before iteration.

First, select the appropriate $H_{1}$ to make the first column of $H_{1} \mathrm{AH}_{1}$ have as many zero elements as possible (but it must satisfy the number of zero elements less than $n-1$ ); then, select the appropriate $\mathrm{H}_{2}$; the same consideration is given to the $N-1$ order principal subformula, until the $N$ 2 order $H_{n-2}$ is obtained. The householder transformation is to find the nonsingular matrix $Q_{0}=H_{1} H_{2} \ldots H_{n-2}$, s.t

$$
Q_{0}^{T} \cdot A \cdot Q_{0}=H,
$$

which is called upper Hessenberg decomposition of $A$, and $H$ is the upper Hessenberg matrix.

Perform QR iteration on the upper Hessenberg matrix $H$; i.e.,

$$
\left\{\begin{array}{l}
H=Q \cdot R \\
\tilde{H}=R \cdot Q
\end{array}\right.
$$

and because of the special format of $H$, we can use the given transformation replacing $\mathrm{QR}$ iterative. In this way, the householder transform is carried out first, and then the given transform is performed. Finally, all eigenvalues of the matrix can be obtained.

Based on the PBH rank condition, the degree of controllability for a given network can be converted as follows:

$$
n=\frac{N_{D}}{N}=\frac{\max _{i}\left\{\mu\left(\lambda_{i}\right)\right\}}{N} .
$$

2.4. The Pricing Controller Location Problem on the FractionalOrder Transportation Networks. At present, there are two typical controllers: one is the traffic signal controller, which influences the vehicles passing through the links by imposing constraints on the natural behavior of the system; the other is the pricing controller, which directly affects the network travel cost and the number of vehicles passing through these links by collecting tolls on the links of a given network. But in this paper the number and installation of the pricing controller are discussed.

According to the $\mathrm{PBH}$ rank condition, we know that the minimum number of driver nodes is equal to the maximum geometric multiplicity of all eigenvalues of the state matrix A. As mentioned in Section 2.2, the elements $a_{i j}$ can be viewed as the ratio of congestion, where $0<a_{i j}(k) \leq 1$. From the perspective of structural controllability theory, our focus is not on the exact value of the element $a_{i j}$, but on whether there is a connection between nodes for given fractionalorder transportation networks. The capture of state matrix mainly focuses on the relationship between nodes and whether the exact value of the elements $a_{i j}$ will collapse to zero; that is, there is no connection between two nodes. The exact framework of fractional-order transportation networks control theory is established based on the fact that the $\mathrm{PBH}$ condition is not related to the current network congestion; also all links in the network are in a stable state in discrete time, regardless of congestion or not.

According to the given network, the connection matrix between nodes is obtained: If node $i$ is the upstream of node $j$, the element $s_{i j}$ will be equal to 1 ; also $s_{i j}=0$ means that two adjacent nodes are not directly connected. The controllable structure shows that our focus should not be on the exact value of $A$, but on the incidence of node connections, so the definition of state matrix $A$ is as follows:

$$
A=d * S(0<d \leq 1) \text {. }
$$

The QR iterative algorithm is used to obtain all eigenvalues $\lambda_{i}$ of state matrix $A$ and their corresponding eigenvectors. The corresponding geometric multiplicity is obtained by $N-\operatorname{rank}\left(A-\lambda_{i} I_{N}\right)$. Get the maximum geometric multiplicity $M_{\left(\lambda_{i}\right)}$ and return the corresponding eigenvalue $\lambda^{M}$. The minimum number of independent driver nodes is equal to the maximum geometric multiplicity of all eigenvalues of the network matrix.

The minimum number of driving nodes has been obtained, and the next step is to determine the position of the controller. The column canonical form is obtained by column transformation of matrix $\mathrm{C}$, where $C=A-\lambda^{M} I_{N}$. After the column canonical forms are obtained, the linear dependence between rows can be clearly revealed, and the rows that are linearly dependent on other rows correspond to the driving node positions that need to be controlled, so as to ensure that the conditions are met and the fractionalorder transportation networks can be controlled. Note that driver nodes are not unique because they depend on the order in which the basic transformations are implemented, and there are many possible choices for linearly related rows. 
This part mainly gives an accurate framework for the number and location of driving nodes in the fractional-order transportation networks, which will be verified by examples later.

\section{Case Test}

3.1. Case Test 1. In order to verify whether it is feasible to place the controller according to our proposed network controllability method in the performance of the control strategy within the network, we summarize the simulation experiments based on the artificial network in this section. We first consider a six-node artificial network, and the corresponding network diagram is as shown in Figure 3.

As shown in Figure 3, if there is direct connection with two adjacent nodes $S_{i j}=1$, other is filled with zero elements. We can get the incidence matrix $S$ based on the previous knowledge as follows:

$$
S=\left(\begin{array}{llllll}
0 & 0 & 1 & 0 & 0 & 0 \\
0 & 0 & 1 & 0 & 0 & 0 \\
1 & 1 & 0 & 1 & 1 & 1 \\
0 & 0 & 1 & 0 & 0 & 1 \\
0 & 0 & 1 & 0 & 0 & 1 \\
0 & 0 & 1 & 1 & 1 & 0
\end{array}\right)
$$

Here, we can define $d=0.5$ and get the expression of the state matrix $A$

$$
A=d * S\left(\begin{array}{cccccc}
0 & 0 & 0.5 & 0 & 0 & 0 \\
0 & 0 & 0.5 & 0 & 0 & 0 \\
0.5 & 0.5 & 0 & 0.5 & 0.5 & 0.5 \\
0 & 0 & 0.5 & 0 & 0 & 0.5 \\
0 & 0 & 0.5 & 0 & 0 & 0.5 \\
0 & 0 & 0.5 & 0.5 & 0.5 & 0
\end{array}\right),
$$

and based on the above state matrix the QR algorithm is used to obtain all eigenvalues and their corresponding eigenvectors; then the geometric multiplicity is obtained for each eigenvalue and for a given state matrix $A$; the maximum geometric multiplicity is

$$
N_{D}=\max _{i}\left\{\mu\left(\lambda_{i}\right)\right\}=2,
$$

where the $\lambda^{M}=0$, so the matrix $C=A-\lambda^{M} I_{N}$ can be deduced as follows:

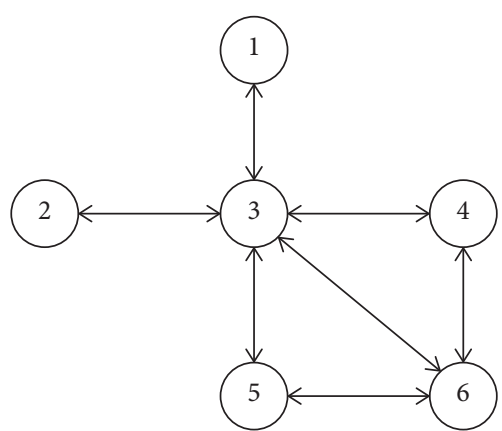

FIGURE 3: Small fractional-order transportation network with 6 nodes.

$$
C=A-0 I_{N}=\left(\begin{array}{cccccc}
0 & 0 & 0.5 & 0 & 0 & 0 \\
0 & 0 & 0.5 & 0 & 0 & 0 \\
0.5 & 0.5 & 0 & 0.5 & 0.5 & 0.5 \\
0 & 0 & 0.5 & 0 & 0 & 0.5 \\
0 & 0 & 0.5 & 0 & 0 & 0.5 \\
0 & 0 & 0.5 & 0.5 & 0.5 & 0
\end{array}\right) .
$$

The simplest form of column is obtained by column transformation of the obtained $C$ matrix. So, the results are shown in Figure 4.

From the eigenvalue of state matrix, we can get $M_{\left(\lambda_{i}\right)}=N_{D}=0$. So, we should at least install two controllers, which make sure that the system satisfies the condition that makes the network controllable. Let $A-\lambda^{M} I_{N}$ be an elementary column transformation and get the column canonical form; it can be seen from Figure 4 that the first column that row $1,2,4,5$ has parameters, so we should randomly select three nodes in nodes $1,2,4,5$ to install controllers, and the installation in the above small network is as shown in the figure. Select one of the two links marked in red and one of the two links marked in green. For the small fractional-order transportation networks, the algorithm of controller location and quantity is completely valid in the small network. However, based on the complexity of fractional-order transportation networks, we need to analyze the feasibility of large-scale network.

3.2. Case Test 2. In this part, we consider larger fractionalorder transportation networks. Our analysis is based on the classic OD network (where the starting node is from node 1 to node 3 ; the end node is from node 26 to node 28 ) in Figure 5, and the nodes in the network are interconnected (if nodes $i$ and nodes $j$ are connected, not only from node $i$ to node $j$, but also from node $j$ to node $i$ ). Here, we call it 
Eigenvalues

$$
C=A-\lambda^{M} I_{N}
$$

Column canonical form

$\left[\begin{array}{l}-1 \\ -0.6715 \\ 0 \\ 0 \\ 0.2647 \\ 1.4068\end{array}\right] \quad M\left(\lambda_{i}\right)=2\left[\begin{array}{cccccc}0 & 0 & 0.5 & 0 & 0 & 0 \\ 0 & 0 & 0.5 & 0 & 0 & 0 \\ 0.5 & 0.5 & 0 & 0.5 & 0.5 & 0.5 \\ 0 & 0 & 0.5 & 0 & 0 & 0.5 \\ 0 & 0 & 0.5 & 0 & 0 & 0.5 \\ 0 & 0 & 0.5 & 0.5 & 0.5 & 0\end{array}\right] \longrightarrow\left[\begin{array}{cccccc}0 & 0 & 1 & 0 & 0 & 0 \\ 0 & 0 & 1 & 0 & 0 & 0 \\ 1 & 0 & 0 & 0 & 0 & 0 \\ 0 & 0 & 0 & 0 & 0 & 1 \\ 0 & 0 & 0 & 0 & 0 & 1 \\ 0 & 0 & 0 & 0 & 1 & 0\end{array}\right]$

FigURE 4: Eigenvalues and their corresponding matrix transformation process.

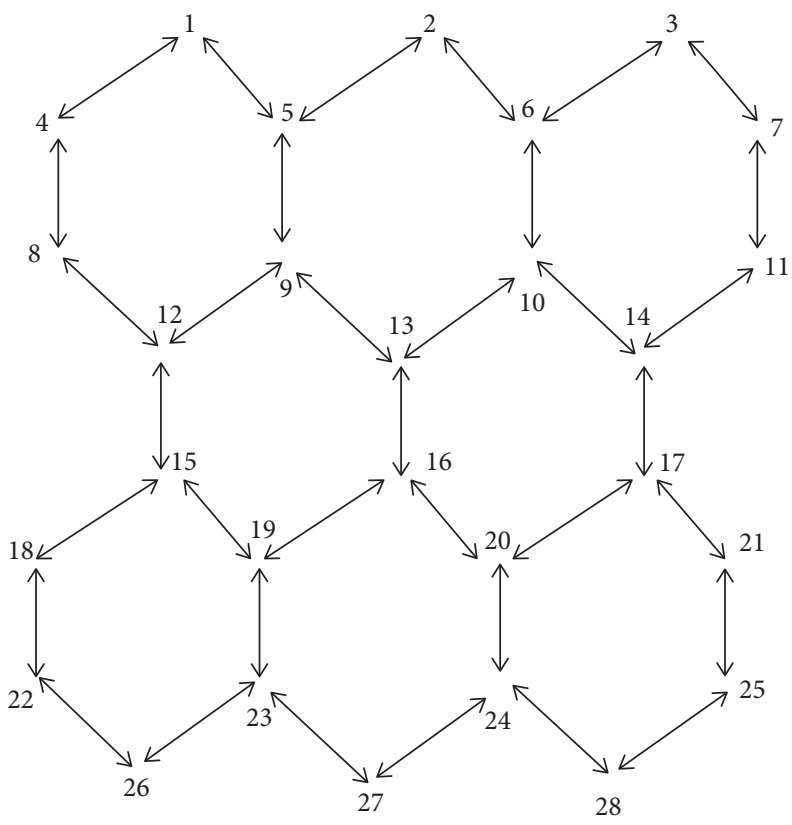

Figure 5: OD networks. 
bidirectional direction. Similarly, we need to get the adjacent matrix $S$ according to the network topology shown in Figure 5, giving the result as follows:

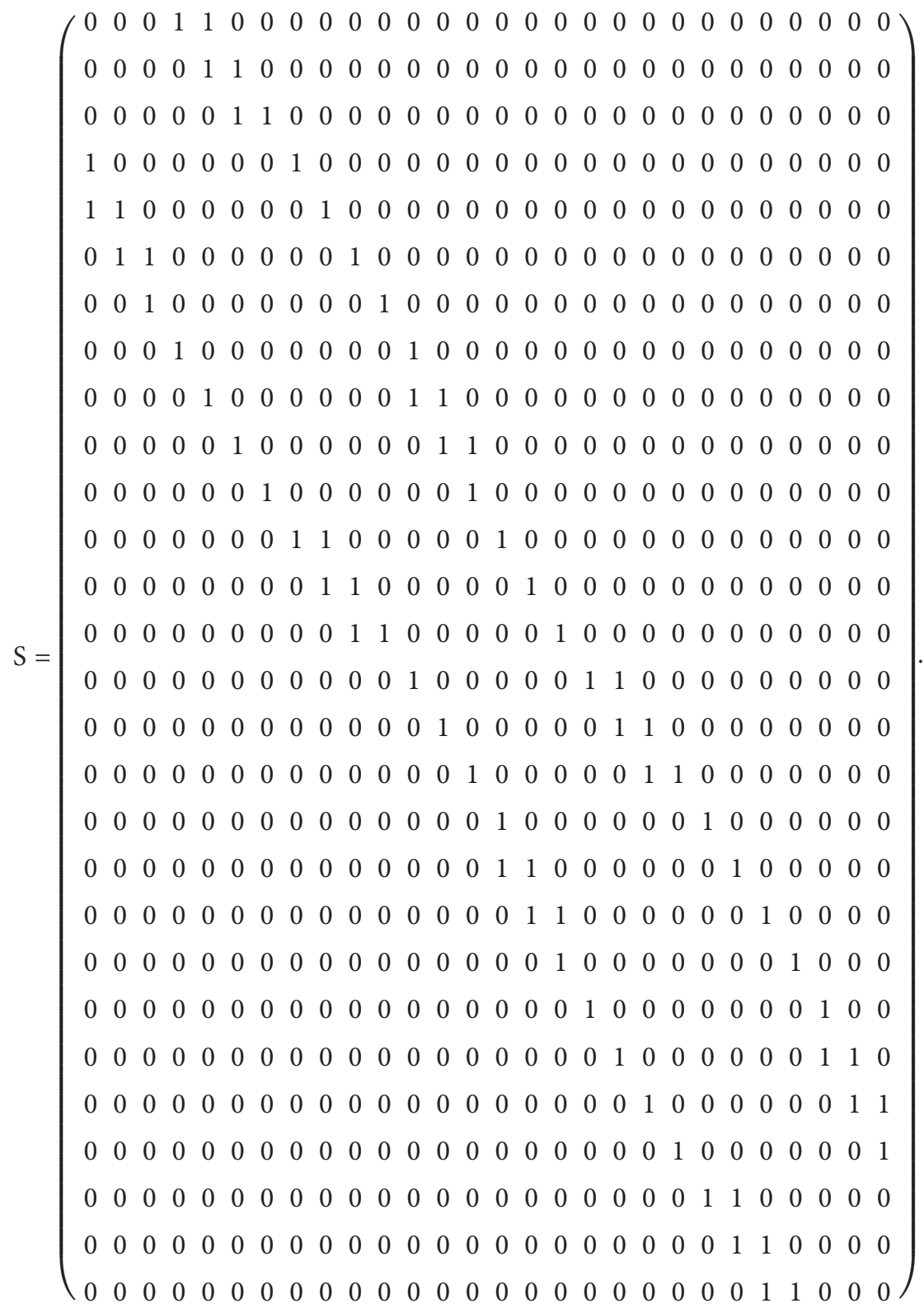

Here, we still define $d=0.5$ and get the expression of the state matrix $A=d * S$; then the eigenvalues are obtained by the QR method and the maximum geometric multiplicities are obtained:

$$
N_{D}=\max _{i}\left\{\mu\left(\lambda_{i}\right)\right\}=2\left(\text { where } \lambda^{M}=1\right) .
$$

The eigenvalue corresponding to the maximum geometric multiplicity is substituted into the matrix $C=A-$ $\lambda^{M} I_{N}$ and transformed into the column canonical form. Here, there is a problem that the method of column transformation for matrix is not unique, which will lead to a little change in the final result. Because the complexity of large fractional-order network does not listen to the method of realizing the basic transformation sequence, there is no connection with the installation position of the driving node which is linearly dependent on other rows in the original topology structure. At this time, we need to filter the unrelated nodes to get the final position where we need to install the controller, so as to ensure that the conditions are met and make the fractional-order transportation system controllable. 


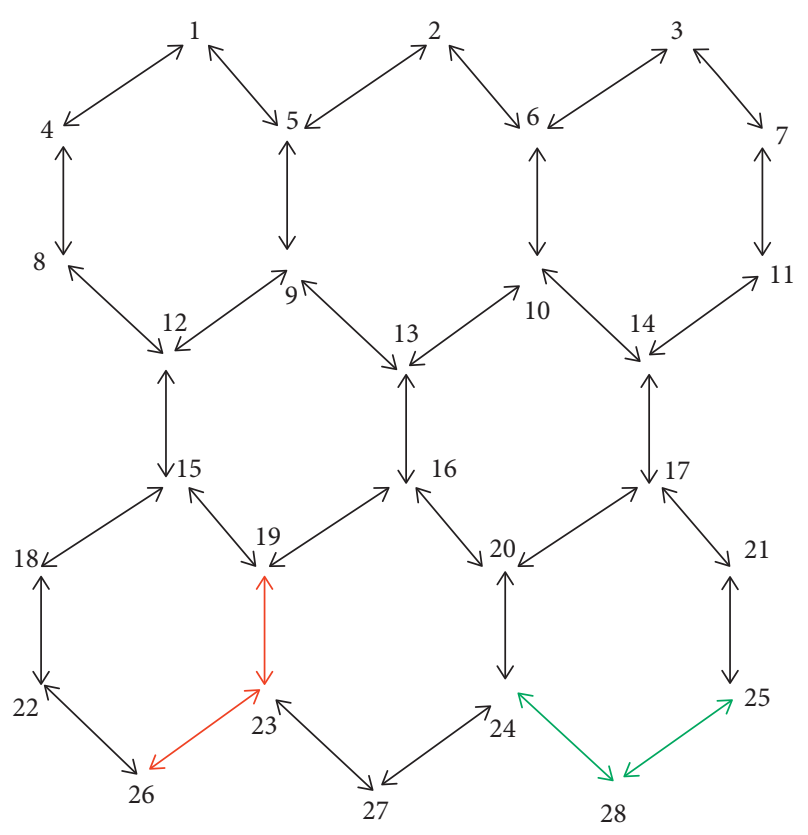

FIgURE 6: The result of OD networks. We need to install two controllers to make the system controllable, and the corresponding positions are as follows: link 19,23 or link 26,23 ; link 24,28 or link 25,28 .

The interception matrix obtained by substituting the maximum multiplicity eigenvalue and column transformation is

$$
A=\left(\begin{array}{ccccccc} 
& 23 & 24 & 25 & 26 & 17 & 28 \\
19 & 1 & 0 & 0 & 0 & 0 & 0 \\
20 & 0 & 1 & 0 & 0 & 0 & 0 \\
21 & 0 & 0 & 1 & 0 & 0 & 0 \\
22 & 0 & 0 & 0 & 1 & 0 & 0 \\
23 & 0 & 0 & 0 & 0 & 1 & 0 \\
24 & 0 & 0 & 0 & 0 & 0 & 1 \\
25 & 0 & 0 & 0 & 0 & 0 & 1 \\
26 & 1 & 0 & 0 & 0 & 0 & 0 \\
27 & 0 & 0 & 0 & 0 & 0 & 0 \\
28 & 0 & 0 & 0 & 0 & 0 & 0
\end{array}\right) .
$$

We can see the linear dependence between rows, so the final result diagram also confirms that for the OD network diagram like Figure 5 we need to install two controllers to make the system controllable, and the corresponding positions are as follows: $\operatorname{link}_{19,23}$ or $\operatorname{link}_{26,23} ; \operatorname{link}_{24,28}$ or $\operatorname{link}_{25,28}$. The results are shown in Figure 6.

Remark 5. The nodes in Figures 1-3 and 7 can be regarded as symbolic geographical locations in the transportation networks, while the links mark the road infrastructure between the two locations. The direction of the link can represent who is the upstream of the vehicle flow in two nodes. Figure (4) shows the eigenvalue and its maximum geometric multiplicity of case test 1 and substitutes it into

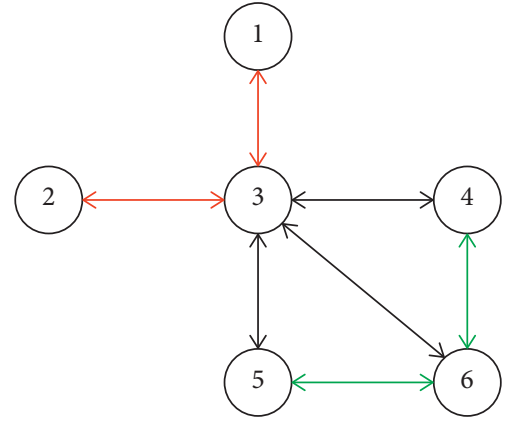

FIgURE 7: Small fractional-order transportation network with 6 nodes. $\mu\left(\lambda^{M}\right)=2$ means that we should at least install two controllers; one of the two links is highlighted in red and green is selected to install the pricing controller.

the matrix for simplification to obtain column canonical form. And Figures 5 and 6 show the classic $O D$ network and its controller result diagram.

\section{Conclusion}

In this work, we study the controllability of the flow-conservation transportation networks with fractional-order dynamics and the location and number of controllers based on the controllability of a complex network with flow conservation.

In order to achieve this goal, we introduce the Caputo fractional derivative on the basis of the flow-conservation complex system to obtain the flow-conservation transportation networks with fractional-order dynamics. The basic modeling assumptions required for the fractionalorder transportation networks to correctly capture the traffic behavior in terms of vehicle propagation and route choice are explained in detail. The relationship between the $\mathrm{cu}-$ mulative number of vehicles, the weight matrix, and the controller matrix and its influence is given.

In traditional control theory, the Kalman rank condition is the main discriminant method. But in many cases, classical controllability theory cannot be used to judge, especially in systems with large sizes or unknown parameters. Based on the exact control theory, the PBH rank condition is proposed to be applicable to arbitrary structure and link weight distribution. Therefore, the controllability research of the fractional-order transportation networks mainly adopts the PBH rank condition; that is, the number of driver nodes is equal to the maximum geometric multiplicity of the eigenvalues of the state matrix, and the installation controller position matrix is obtained through transformation. However, in practical applications, the traditional eigenvalue method has the problem of insufficient accuracy when the matrix order is large. Therefore, this paper introduces QR iteration to obtain the system algorithm of the number and location of the fractional-order transportation networks controller.

Finally, this paper analyzes the effectiveness through two cases. In a small network with fewer nodes, the number and location of controllers have obtained accurate results. On the 
basis of structural theory, using our algorithm can also get the number and location of the controller of the second OD network, which shows that this is feasible for the fractional traffic system.

\section{Data Availability}

There are no publicly archived datasets utilized in this paper.

\section{Conflicts of Interest}

The authors declare that they have no conflicts of interest.

\section{Acknowledgments}

This work was supported in part by the Science and Technology Research Program of Education Commission of Chongqing (Grant KJZD-M20200070) and in part by the Team Building Project for Graduate Tutors in Chongqing (Grant JDDSTD201802).

\section{References}

[1] Y.-Y. Liu, J.-J. Slotine, and A.-L. Barabási, "Controllability of complex networks,” Nature, vol. 473, no. 7346, pp. 167-173, 2011.

[2] Z. Yu, "Controllability gramian and kalman rank condition for mean-field control systems," ESAIM: Control, Optimisation and Calculus of Variations, vol. 27, 2021.

[3] G. Joseph and C. R. Murthy, "Controllability of linear dynamical systems under input sparsity constraints," 2020, https://arxiv.org/abs/1912.12224.

[4] L. Wang, G. Zhao, and Z. Kong, "Controllability and optimization of complex networks based on bridges," Complexity, vol. 2020, Article ID 6695026, 10 pages, 2020.

[5] X. Wang and L. Xiang, "Optimizing network controllability with minimum cost," Complexity, vol. 2021, Article ID 6657307, 13 pages, 2021.

[6] M. Dotoli, M. P. Fanti, and C. Meloni, "A signal timing plan formulation for urban traffic control," Control Engineering Practice, vol. 14, no. 11, pp. 1297-1311, 2006.

[7] S. Ukkusuri, K. Doan, and H. M. A. Aziz, "A bi-level formulation for the combined dynamic equilibrium based traffic signal control," Procedia-Social and Behavioral Sciences, vol. 80, no. 1, pp. 729-752, 2013.

[8] M. Rinaldi, C. M. J. Tampère, and F. Viti, "On characterizing the relationship between route choice behavior and optimal traffic control solution space," Transportation Research Procedia, vol. 23, pp. 700-719, 2017.

[9] G. Bianchin and F. Pasqualetti, "Gramian-based optimization for the analysis and control of traffic networks," IEEE Transactions on Intelligent Transportation Systems, vol. 21, no. 7, pp. 3013-3024, 2020.

[10] M. Rinaldi, "Controllability of transportation networks," Transportation Research Part B: Methodological, vol. 118, pp. 381-406, 2018.

[11] B. Haci, M. Toufik, and H. Zakia, "Active control of a chaotic fractional order economic system," Entropy, vol. 17, no. 8, pp. 5771-5783, 2015.

[12] J. Wang, D. Xu, and J. Zhou, "Fractional-order modeling and analysis of a variable structure hybrid energy storage system for EVs," Complexity, vol. 2020, Article ID 7643812, 10 pages, 2020.
[13] Z. Hammouch and T. Mekkaoui, "Control of a new chaotic fractional-order system using mittag-leffler stability," Nonlinear Studies, vol. 22, no. 4, pp. 1-13, 2015.

[14] W. Zou and Z. Xiang, "Containment control of fractionalorder nonlinear multi-agent systems under fixed topologies," IMA Journal of Mathematical Control and Information, vol. 35, 2018.

[15] S. Huang and Z. Xiang, "Stability of a class of fractional-order two-dimensional non-linear continuous-time systems," IET Control Theory \& Applications, vol. 10, 2016.

[16] K. M. Owolabi and Z. Hammouch, "Spatiotemporal patterns in the Belousov-Zhabotinskii reaction systems with Atangana-Baleanu fractional order derivative," Physica A: Statistical Mechanics and Its Applications, vol. 523, 2019.

[17] T. Mekkaoui, H. Zakia, and D. Kumar, A New Approximation Scheme for Solving Ordinary Differential Equation with Gomez-Atangana-Caputo Fractional Derivative, CRC Press, Boca Raton, FL, USA, 2019.

[18] H. Zhang, Y. D. Chen, and B. B. Xu, "Controllability of fractional-order directed complex networks," Modern Physics Letters B, vol. 28, no. 27, p. 143006, 2014.

[19] H. Zhang and H. Zhou, "Controllability of fractional-order directed complex networks, with self loop and double edge structure," Journal of Circuits, Systems and Computers, vol. 24, 2015.

[20] K. Balachandran, V. Govindaraj, L. Rodríguez-Germa, and J. J. Trujillo, "Controllability results for nonlinear fractionalorder dynamical systems," Journal of Optimization Theory and Applications, vol. 156, no. 1, pp. 33-44, 2013.

[21] Z. Yuan, C. Zhao, Z. Di, W. X Wang, and Y. C Lai, "Exact controllability of complex networks," Nature Communications, vol. 4, no. 2447, p. 2447, 2013.

[22] Z. Zhong and C. Yuan, "Exact controllability of multiplex networks," New Journal of Physics, vol. 16, no. 10, p. 103036, 2014.

[23] R. E. Kalman, "Mathematical description of linear dynamical systems," Journal of the Society for Industrial and Applied Mathematics Series A Control, vol. 1, no. 2, pp. 152-192, 1963.

[24] G. Mei, X. Wu, D. Ning, and J.-A. Lu, "Finite-time stabilization of complex dynamical networks via optimal control," Complexity, vol. 21, pp. 417-425, 2016.

[25] T. Summers, F. Cortesi, and J. Lygeros, "On submodularity and controllability in complex dynamical networks," IEEE Transactions on Control of Network Systems, vol. 3, no. 1, pp. 91-101, 2014.

[26] A. Olshevsky, "Minimal controllability problems," IEEE Transactions on Control of Network Systems, vol. 1, no. 3, pp. 249-258, 2014.

[27] X. Ma, H. Zhao, and B. Wang, "Controllability of deterministic networks with the identical degree sequence," PLoS One, vol. 10, no. 5, p. e0127545, 2015.

[28] L.-Z. Wang, Y.-Z. Chen, W.-X. Wang, and Y.-C. Lai, "Physical controllability of complex networks," Scientific Reports, vol. 7, no. 1, p. 40198, 2017.

[29] T. Bai, L. I. Shaoyuan, and Y. Zou, “"Minimum input selection of reconfigurable architecture systems for structural controllability," Sciece China," Information Sciences, vol. 62, no. 1, pp. 223-225, 2019.

[30] V. Webster, "Traffic signal settings," Road Research Technical Paper, vol. 39, 1958.

[31] Y. Sheffi, Urban Transportation Networks: Equilibrium Analysis with Mathematical Programming Methods, PrenticeHall, Hoboken, NJ, USA, 1984. 
[32] G. E. Cantarella and A. Sforza, Network Design Models and Methods for Urban Traffic Management, Springer, Berlin, Germany, 1995.

[33] N. Bekiaris-Liberis, C. Roncoli, and M. Papageorgiou, "Highway traffic state estimation per lane in the presence of connected vehicles," Transportation Research Part B: Methodological, vol. 106, pp. 1-28, 2017.

[34] S. Jia, H. Peng, and S. Liu, "Urban traffic state estimation considering resident travel characteristics and road network capacity," Journal of Transportation Systems Engineering and Information Technology, vol. 11, no. 5, pp. 0-85, 2011.

[35] Y. ming, "Traffic state estimation for urban road networks using a link queue model," Transportation Research Record, vol. 2623, no. 1, pp. 29-39, 2017.

[36] K. Boel and K. Rene, "Parameter estimation for stochastic hybrid model applied to urban traffic flow estimation," IET Control Theory \& Applications, vol. 9, 2015.

[37] N. Bekiaris-Liberis, C. Roncoli, and M. Papageorgiou, "Highway traffic state estimation with mixed connected and conventional vehicles," IEEE Transactions on Intelligent Transportation Systems, vol. 17, no. 12, pp. 3484-3497, 2016.

[38] S. Contreras, P. Kachroo, and S. Agarwal, "Observability and sensor placement problem on highway segments: a traffic dynamics-based approach," IEEE Transactions on Intelligent Transportation Systems, vol. 17, no. 3, pp. 848-858, 2016.

[39] M. Rinaldi, W. Himpe, and C. M. J. Tampère, "A sensitivitybased approach for adaptive decomposition of anticipatory network traffic control," Transportation Research Part C: Emerging Technologies, vol. 66, no. 2, pp. 150-175, 2016.

[40] Z. Huide, R. Bouyekhf, and A. E. Moudni, "Modelling and Ho control of urban transportation network," IFAC Proceedings Volumes, vol. 45, no. 24, pp. 72-77, 2012.

[41] M. Zhou, S. Cheng, L. Xu, L. Wang, Q. Guo, and W. Cai, "Robust switching Gain-Based fractional-order sliding mode control for wind-powered microgrids," Complexity, vol. 2021, no. 6, Article ID 6697792, 12 pages, 2021.

[42] D. Zhao and J. Mao, "New controllability results of fractional nonlocal semilinear evolution systems with finite delay," Complexity, vol. 2020, Article ID 7652648, 13 pages, 2020.

[43] M. Ghasemi and K. Nassiri, "On controllability of fractional continuous-Time systems," Mathematical Problems in Engineering, vol. 2021, Article ID 5557068, 14 pages, 2021.

[44] C. Zhao, A. Zeng, R. Jiang, Z. Yuan, and W. X. Wang, "Controllability of flow-conservation networks," Physical Review, vol. 96, no. 1, p. 12314, 2017. 